\title{
Extremely low birth weight infants supplemented with probiotic Lactobacillus reuteri DSM 17938 had higher gut microbiota diversity during the neonatal period
}

\author{
Jie Xu1 ${ }^{1}$, Maria Tärnberg ${ }^{1}$, Erik Wejryd ${ }^{1,2}$, Giovanna Marchini ${ }^{3}$, Baldvin Jonsson ${ }^{3}$, Eva \\ Sverremark Ekström ${ }^{4}$, Magalí Martí ${ }^{1}$, Maria Jenmalm ${ }^{1}$ and Thomas Abrahamsson ${ }^{1,2}$
}

${ }^{1}$ Department of Clinical and Experimental Medicine and ${ }^{2}$ Department of Paediatrics, Linköping University, Linköping, Sweden, ${ }^{3}$ Astrid Lindgren Children's Hospital, Karolinska University Hospital, Karolinska Institute, Department of Neonatology, Stockholm, Sweden ${ }^{4}$ Department of Molecular Biosciences, The Wenner-Gren Institute, Stockholm University, Stockholm, Sweden

\section{Background and aims}

Dysbiosis, with a reduced microbial diversity, has been associated with multiple diseases, including necrotizing enterocolitis, in premature infants. It is hypothesized that probiotics may counteract the dysbiosis and hence promote health. The aim of the current study was to investigate the effect of probiotic supple-mentation on the gut microbiota in extremely low birth weight (ELBW) infants.

\section{Methods}

In a randomized, double-blind, multicenter, placebo-controlled trial, ELBW infants with a birth weight $<1000 \mathrm{~g}$, born at gestational week $<28$, were supplemented with either probiotics ( $L$. reuteri DSM 17938) or placebo ( $n=67$ and 65 , respectively) from birth until gestational week 36 . A total of 551 stool samples were used for profiling the gut microbiota with next generation sequencing of the 16 s rRNA gene at $1(n=54 / 54), 2(n=54 / 53), 3(n=51 / 51)$ and 4 $(n=52 / 48)$ weeks of age, at gestational week $36(n=50 / 37)$ and at 2 -years $(n=20 / 27)$ of age.
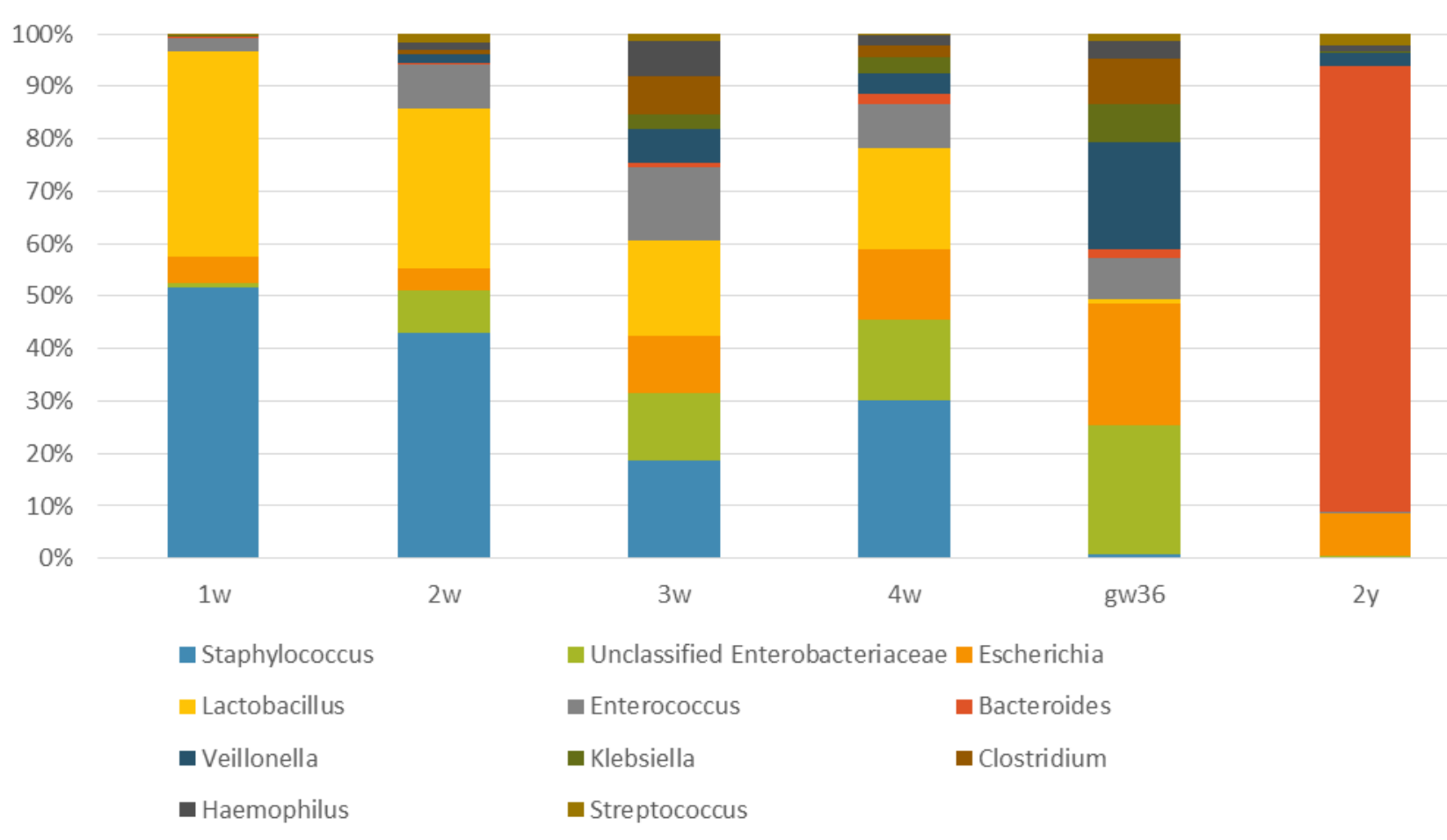

Figure 1A. Average abundance of bacterial genera in the probiotics group.

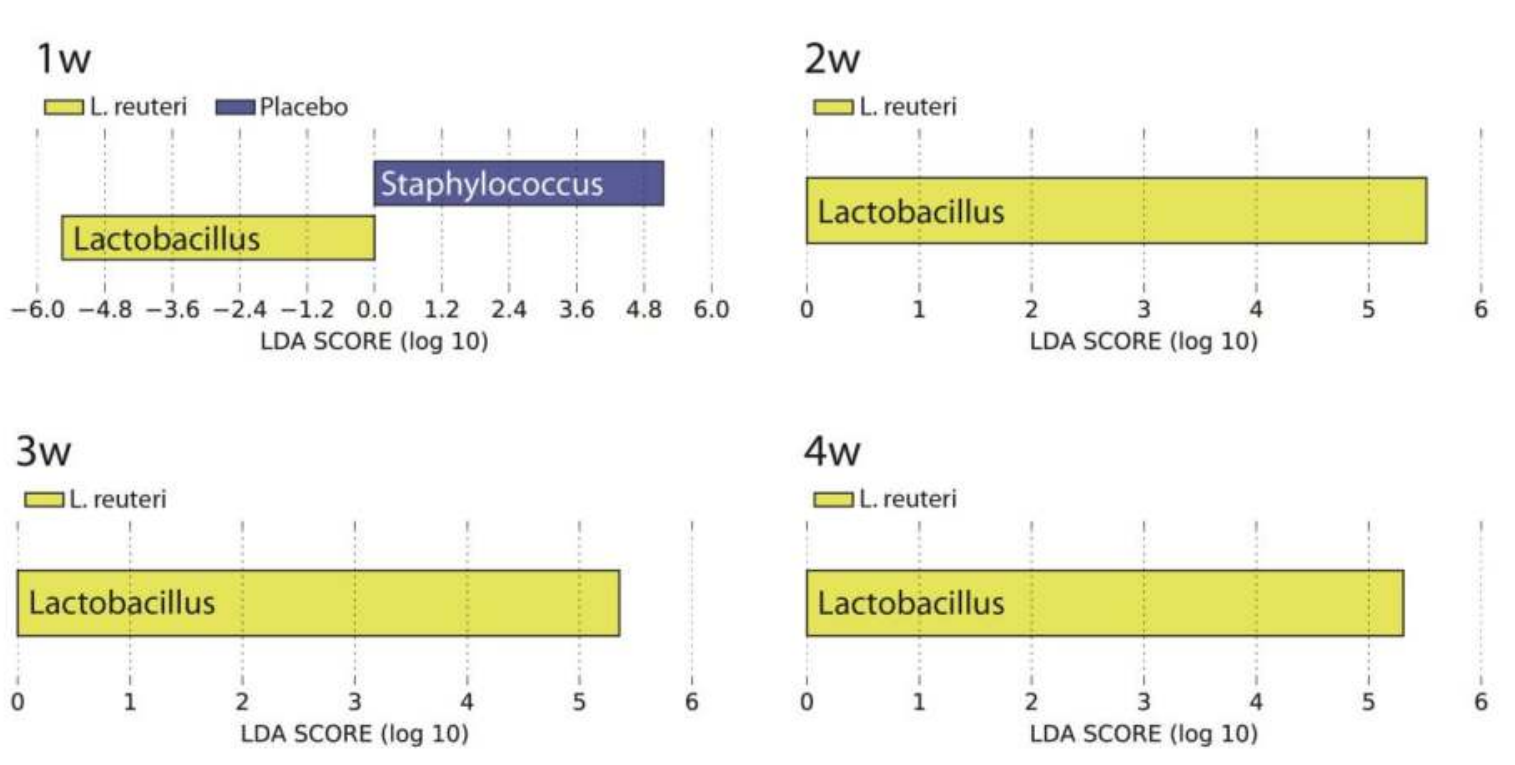

Figure 2. Linear discriminant analysis effect size, showing bacterial taxa that significantly differed in abundance between groups.

Acknowledgements

The study was supported by the Swedish Research Council, Swedish Society for Medical Research and BioGaia AB, Sweden.

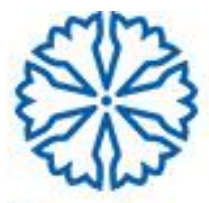

Region Östergötland

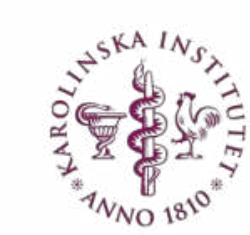

Karolinska Institutet

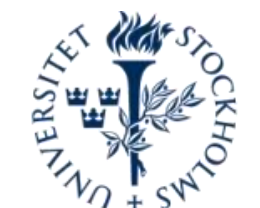

Stockholm University
Demographic data

gestational age at birth, weeks, median (IQR)

birth weight, grams, median (IQR)

girls, $\mathrm{n}(\%)$

infants from multiple pregnancy, $\mathrm{n}(\%)$

Perinatal factors, $n(\%)$

premature rupture of membranes

chorioamnionitis

Caesarean section

maternal antibiotics

Clinical outcomes, $n(\%)$

onset of NEC during the study period

onset of sepsis during the study period

Antibiotic treatment and mechanical ventilation

antibiotics 1 st week of life, $n(\%)$

antibiotics 2 nd week of life, $n(\%)$

antibiotics 3rd week of life, $n(\%)$

antibiotics 4 th week of life, $n(\%)$

antibiotics until end of gw36, days, median (IQR)

mechanical ventilation, days, median (IQR)

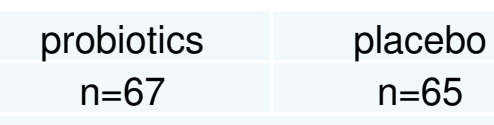

$25.7(24.6-26.4) 25.7(24.6-26.5)$

$726(630-814) \quad 760(615.5-864)$

$36(54) \quad 23(35)$

$28(42) \quad 19(29)$

$20(30) \quad 10(15)$

$49(73) \quad 37(57)$

$41(61) \quad 31(48)$

$7(10) \quad 8(12)$

$38(57) \quad 34(52)$

$57(85) \quad 50(77)$

$48(72) \quad 44(68)$

$49(73) \quad 39(60)$

$26(19-37) \quad 26(12.5-37.5)$

$14(6-31) \quad 15(4-33)$
$24(36) \quad 16(25)$

$67(100) \quad 65(100)$

Table 1. Background data.

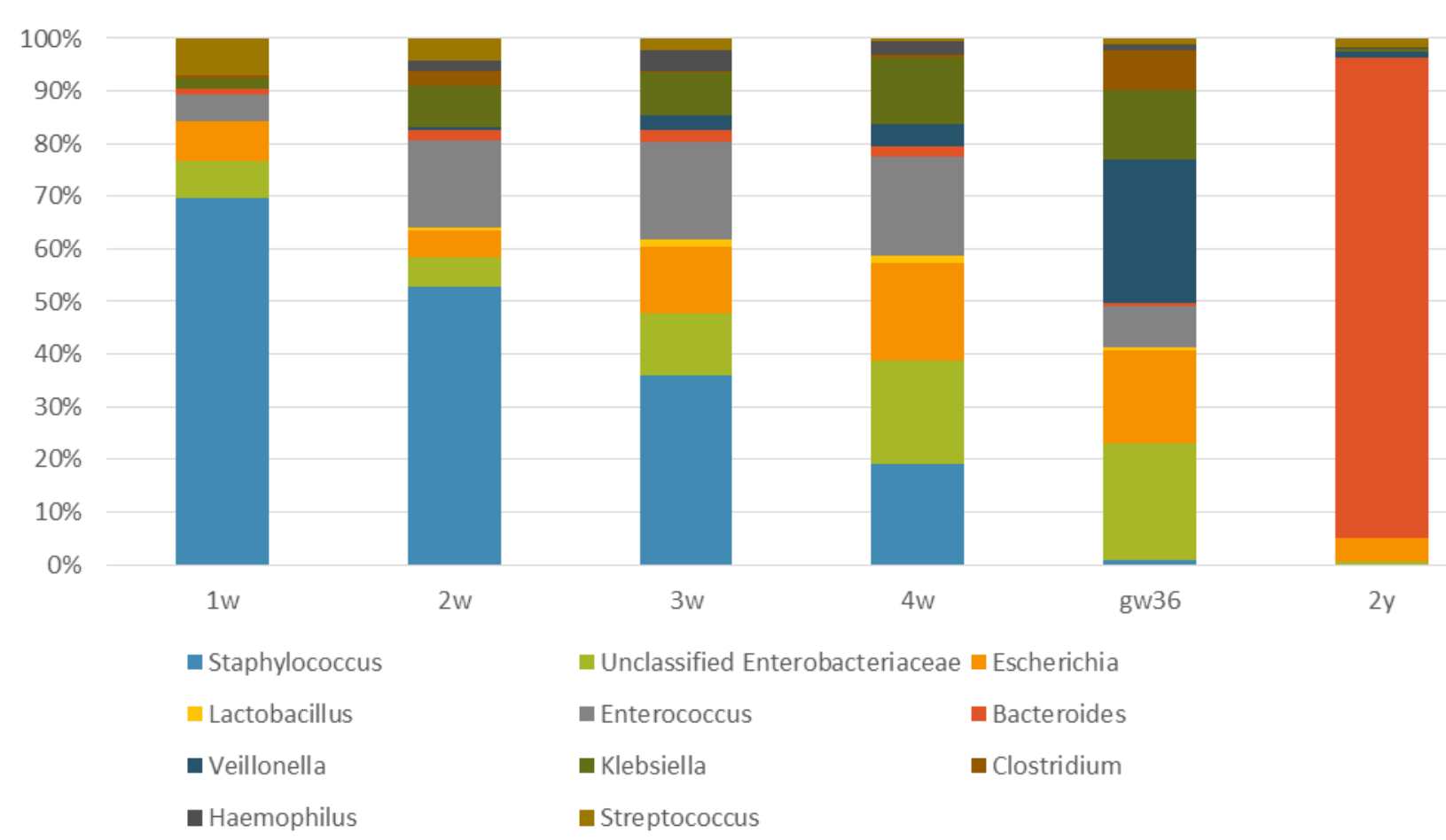

Figure 1B. Average abundance of bacterial genera in the placebo group.

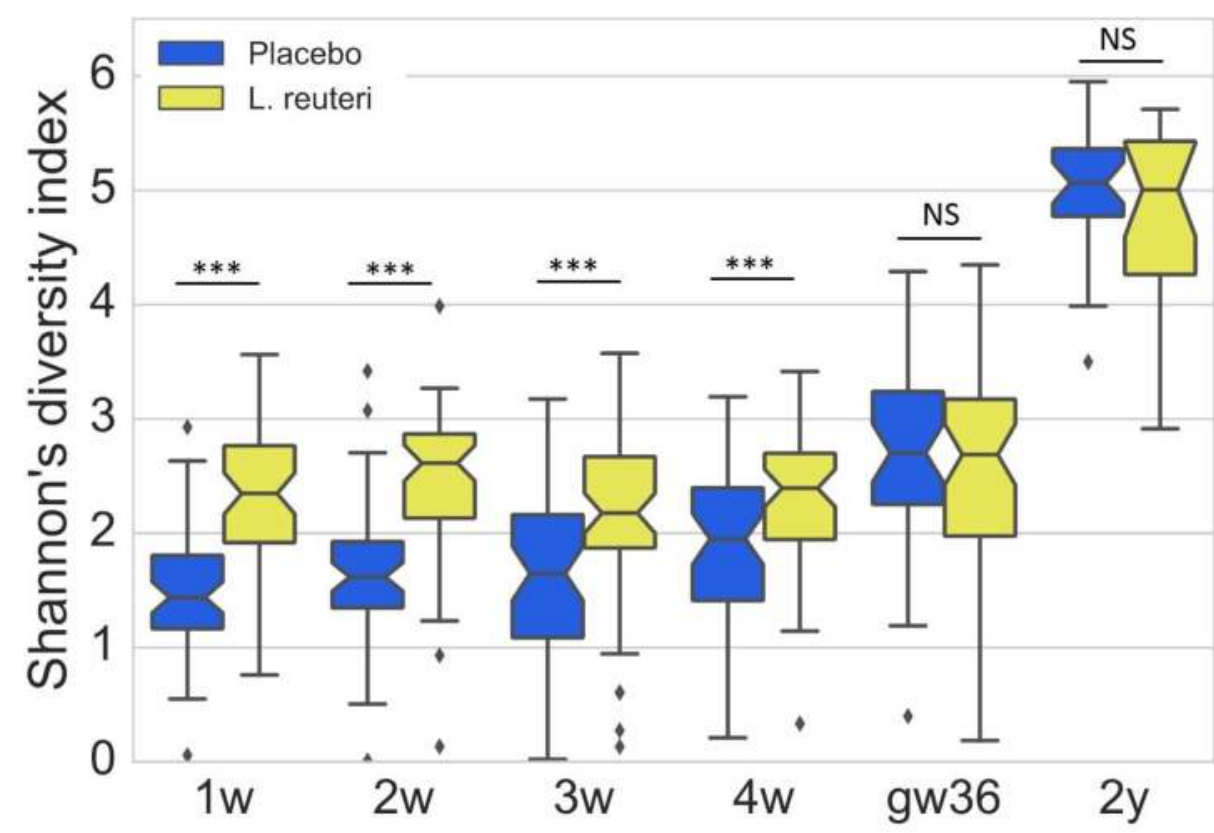

Figure 3 Alpha-diversity, in terms of Shannon's index of diversity.

\section{Conclusions}

The main finding of this study is that supplementation of L. reuteri DSM 17938, in ELBW preterm infants, affects the microbiome during the neonatal period in terms of altered composition and increased alpha-diversity. 